\title{
Protein Dynamics in a Broad Frequency Range: Dielectric Spectroscopy Studies
}

\author{
Masahiro Nakanishi ${ }^{1}$ and Alexei P. Sokolov ${ }^{2,3, *}$ \\ ${ }^{1}$ Department of Applied Physics, The Hebrew University of Jerusalem, Givat Ram, Jerusalem \\ 91904, Israel \\ ${ }^{2}$ Department of Chemistry, and Joint Institute for Neutron Sciences, University of Tennessee, \\ Knoxville, TN 37996, USA \\ ${ }^{3}$ Chemical Sciences Division, Oak Ridge National Laboratory, Oak Ridge, TN 37831, USA \\ *Corresponding author: sokolov@utk.edu; +1-865-974-3852; 1420 Circle Dr., Knoxville, TN \\ 37996-1600, USA
}

\begin{abstract}
We present detailed dielectric spectroscopy studies of dynamics in two hydrated proteins, lysozyme and myoglobin. We emphasize importance of explicit account for possible MaxwellWagner (MW) polarization effects in protein powder samples. Combining our data with earlier literature results, we demonstrate the existence of three major relaxation processes in globular proteins. To understand the mechanisms of these relaxations we involve literature data on neutron scattering, simulations and NMR studies. The faster process is ascribed to coupled protein - hydration water motions and has relaxation time $\sim 10-50 \mathrm{ps}$ at room temperature. The intermediate process is $\sim 10^{2}-10^{3}$ times slower than the faster process and might be strongly affected by MW-polarizations. Based on analysis of data obtained by different experimental techniques and simulations, we ascribe this process to large scale domain-like motions of
\end{abstract}


proteins. The slowest observed process is $\sim 10^{6}-10^{7}$ times slower than the faster process and has anomalously large dielectric amplitude $\Delta \varepsilon \sim 10^{2}-10^{4}$. The microscopic nature of this process is not clear, but it seems to be related to the glass transition of hydrated proteins. The presented results suggest a general classification of the relaxation processes in hydrated proteins.

\section{Keywords}

protein dynamics; hydration water; glass transition; dielectric spectroscopy; lysozyme, myoglobin

\section{PACS}

64.70.P- Glass transitions of specific systems; 87.14.E- Proteins; 87.15.H- Dynamics of biomolecules

\section{Abbreviations}

Broadband Dielectric Spectroscopy (BDS)

Maxwell-Wagner (MW)

Anomalously Intense (AI)

Bovine Serum Albumin (BSA) 


\section{Introduction}

It is well recognized that understanding function and activity of biological macromolecules requires fundamental understanding of their structure and dynamics. While structures of many proteins and nucleic acids are already known, their dynamics remain poorly understood. Nuclear magnetic resonance (NMR) spectroscopy, neutron scattering and molecular dynamic (MD) simulations are actively employed to study microscopic details of protein dynamics [1-13]. They revealed existence of several relaxation processes on $\mathrm{ns}$ - ps time scale, but a general picture of protein dynamics is still absent. Neutron scattering and MD studies are still limited to protein dynamics only on time scales faster than hundreds of ns, while NMR provides very local information and misses global view of the protein dynamics.

In contrast to these techniques, broadband dielectric spectroscopy (BDS) provides measurements of dynamics in extremely broad frequency range with very high accuracy [14]. However, it has significant drawbacks due to the absence of any microscopic information about the motions contributing to the dielectric relaxation spectra. These spectra reflect motions of dipoles and charges, and strong ionic conductivity (is always significant in biological samples) creates significant problem in analysis and interpretation of the BDS spectra. BDS is also insensitive to motions that do not modulate dipole moment of the system. Moreover, intrinsic heterogeneities and interfaces between macromolecules, their complexes and solvent lead to Maxwell-Wagner (MW) polarization effects that create additional relaxation peaks in the dielectric spectra. Nevertheless, active use of BDS in recent years lead to significant advances in our understanding of protein dynamics in a broad frequency and temperature ranges [12, 15-17].

In many cases dynamics of proteins is studied in dilute solutions [15, 18-21], because it helps to isolate dynamics of individual protein molecules. In the case of BDS studies it has several 
disadvantages: (i) No dynamics can be studied at frequencies below the tumbling (rotation of entire molecules), that at ambient conditions in aqueous solution limits the time scale to faster than $\sim 10-100 \mathrm{~ns}[15,18-20,22]$; (ii) Significant contribution from solvent and ionic conductivity complicates the analysis at high and low frequencies, respectively; (iii) Solvent crystallization limits the temperature range of the studies. Dielectric studies of hydrated protein powders help to eliminate some of these problems.

The main goal of the current studies is the analysis and microscopic interpretation of several relaxation processes detected in the dielectric spectra of protein powders. We present detailed BDS studies of two proteins, lysozyme and myoglobin, at different levels of hydration and in a broad temperature range. The results are compared to BDS studies of other proteins and to studies of lysozyme and myoglobin by other experimental techniques and MD simulations. Based on our analysis we formulate microscopic interpretation of major relaxation processes detected in the dielectric spectra of protein powders. The analysis also identifies some misinterpretations and artifacts in earlier BDS studies of protein powders.

\section{Experimental}

\section{Sample Preparation}

Lyophilized powders of lysozyme from chicken egg white, with residual buffer salts $\leq 10$ $\%$ (L4919, Sigma-Aldrich Co. LLC.) and myoglobin from equine skeletal muscle, 95 - $100 \%$ assay (M0630, Sigma-Aldrich Co. LLC.), were purchased and used without any further purification. Desired hydration level, $h=$ (weight of water)/(weight of protein), was achieved by keeping lyophilized protein powders above an open water in a desiccator. Final hydration was 
estimated using weighing the sample before and after hydration process. Protein powder was pressed into pellets about $1 \mathrm{~mm}$ of thickness and $20 \mathrm{~mm}$ of diameter. It was formed by compressing powder filled in a cylindrical die at about $150 \mathrm{MPa}$ of pressure at room temperature. This compression does not lead to unfolding [23-26], but forms dense samples without significant amount of voids. Removal of voids is important for suppression of MW polarization effects (see the Discussion section).

Apparent density of the initial dried pellet was estimated from measured dimensions and weight. For 8 lysozyme samples and 4 myoglobin samples, the apparent densities were estimated to be $1.05 \pm 0.04 \mathrm{~g} / \mathrm{cm}^{3}$ and $1.06 \pm 0.03 \mathrm{~g} / \mathrm{cm}^{3}$, respectively. These values are consistent with the estimate from the fraction of close packed protein molecules: Partial density of globular protein is reported to be in the range of $1.3-1.5 \mathrm{~g} / \mathrm{cm}^{3}$ regardless of protein species [27-33]. Assuming that the partial density of lysozyme and myoglobin is $\sim 1.4 \mathrm{~g} / \mathrm{cm}^{3}$, their apparent density corresponds to packing fraction of the protein particles $\sim 75 \%$. This value is close to the fraction of random close-packed sphere $64 \%[34,35]$. In reality proteins are not spherical but rather ellipsoidal [36, 37]. In this case, even higher packing fraction approaching that of close-packed hexagonal sphere $74 \%$ is expected [34]. Furthermore, it has been reported that crystals of a variety of small organic molecules and proteins have packing fractions around $75 \%$ [29]. Therefore, it is expected that our protein pellets don't contain significant amount of voids.

\section{Dielectric Spectroscopy}

Complex permittivity spectrum, $\varepsilon^{*}(\omega)=\varepsilon^{\prime}(\omega)-i \varepsilon^{\prime \prime}(\omega)$, was measured by two impedance spectrometers, here $\varepsilon^{\prime}$ is the real part and $\varepsilon^{\prime \prime}$ is the imaginary part of the dielectric permittivity. Fourier response impedance analyzer (Alpha analyzer, Novocontrol) was used in 
the frequency range from $10 \mathrm{mHz}$ to $10 \mathrm{MHz}$. RF-impedance analyzer (E4991A, Agilent technology) was employed in the frequency range from $1 \mathrm{MHz}$ to $3 \mathrm{GHz}$. For the former, parallel plate discs sandwiching sample were used, whereas for the latter specially designed highfrequency electrodes [38] were used. For lower frequency measurements, sample was sealed by Teflon cylinder to avoid losses of water. We stress that no any insulator in between sample and electrodes have been used. It is known that the use of the insulating layers leads to artificial relaxation processes and misinterpretations $[39,40]$. The sample temperature was controlled by Quatro cryosystem (Novocontrol) with measurements going from higher to lower temperatures. Before measurements at any temperature, sample was equilibrated for at least 20 minutes.

\section{Analysis and Fits of the Spectra}

One of the crucial problems in dielectric spectroscopy of highly conductive material is that loss peaks representing dielectric relaxation processes are masked by strong dc-conductivity. Conductivity does not contribute directly to the real part of $\varepsilon^{*}$. However, relaxation process appears only as a smooth step in the real part, while it appears as a distinct peak in the imaginary part. In that case, the derivative analysis of the real part of the spectrum is helpful [41]. It is usually presented as:

$$
\operatorname{Drv}(\omega)=-\frac{1}{2} \cdot \frac{d \varepsilon^{\prime}}{d \ln \omega}
$$

where $\omega$ is angular frequency. Both imaginary part and the derivative spectrum have peaks at the same frequencies, but the derivative spectrum excludes conductivity contribution and peaks are sharper than in the imaginary part [41]. Therefore, lower frequencies are accessible and each component becomes better resolved in the derivative spectrum. 
Figures 1 and 2 present several representative complex permittivity and the derivative spectra for lysozyme at $h=0.36$ and for myoglobin at $h=0.34$, respectively. The spectra of two proteins share similar features: (i) The imaginary part is dominated by strong dc-conductivity, and at $T>190 \mathrm{~K}$ only one loss peak is well resolved at high frequency (e.g. at around $1 \mathrm{MHz}$ at $203 \mathrm{~K}$ in both lysozyme and myoglobin); (ii) Large steps/shoulders with $\Delta \varepsilon \sim 100-3000$ appear in the real part and the derivative spectra at frequencies lower than the peak in $\varepsilon^{\prime \prime}$; (iii) At temperature below $170 \mathrm{~K}$, bimodal peak and the so-called nearly constant loss (NCL) are observed. 


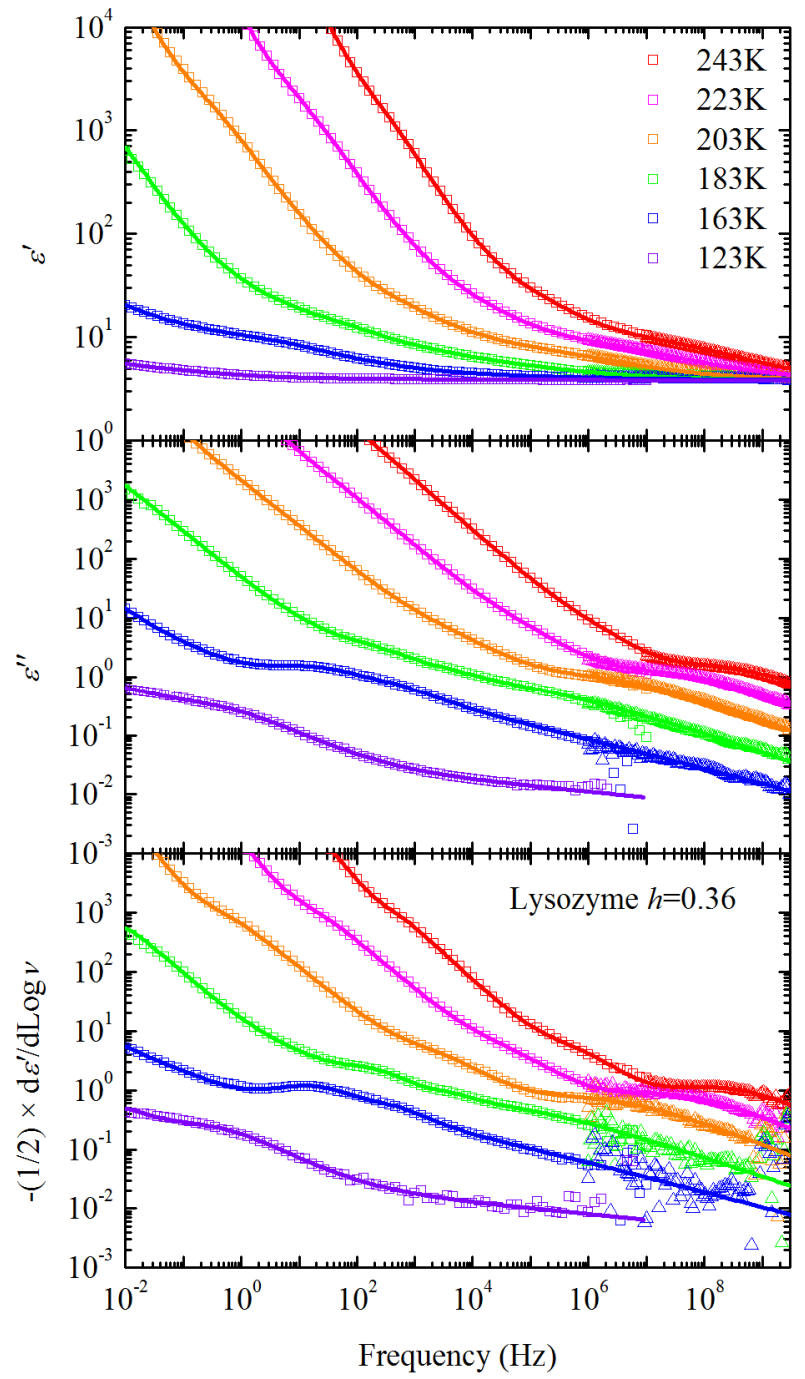

Fig. 1: Complex permittivity spectra, real part (top panel), imaginary part (middle panel), and derivative spectra (bottom panel), of lysozyme at $h=0.36$ at representative temperatures. Symbols show experimental data, and solid lines show fits composed of dc-conductivity, electrode polarization, processes $\mathrm{B}, \mathrm{D}$, and $\mathrm{G}$ (and $\mathrm{E}$ and $\mathrm{F}$ at $T \leq 183 \mathrm{~K}$ ). 


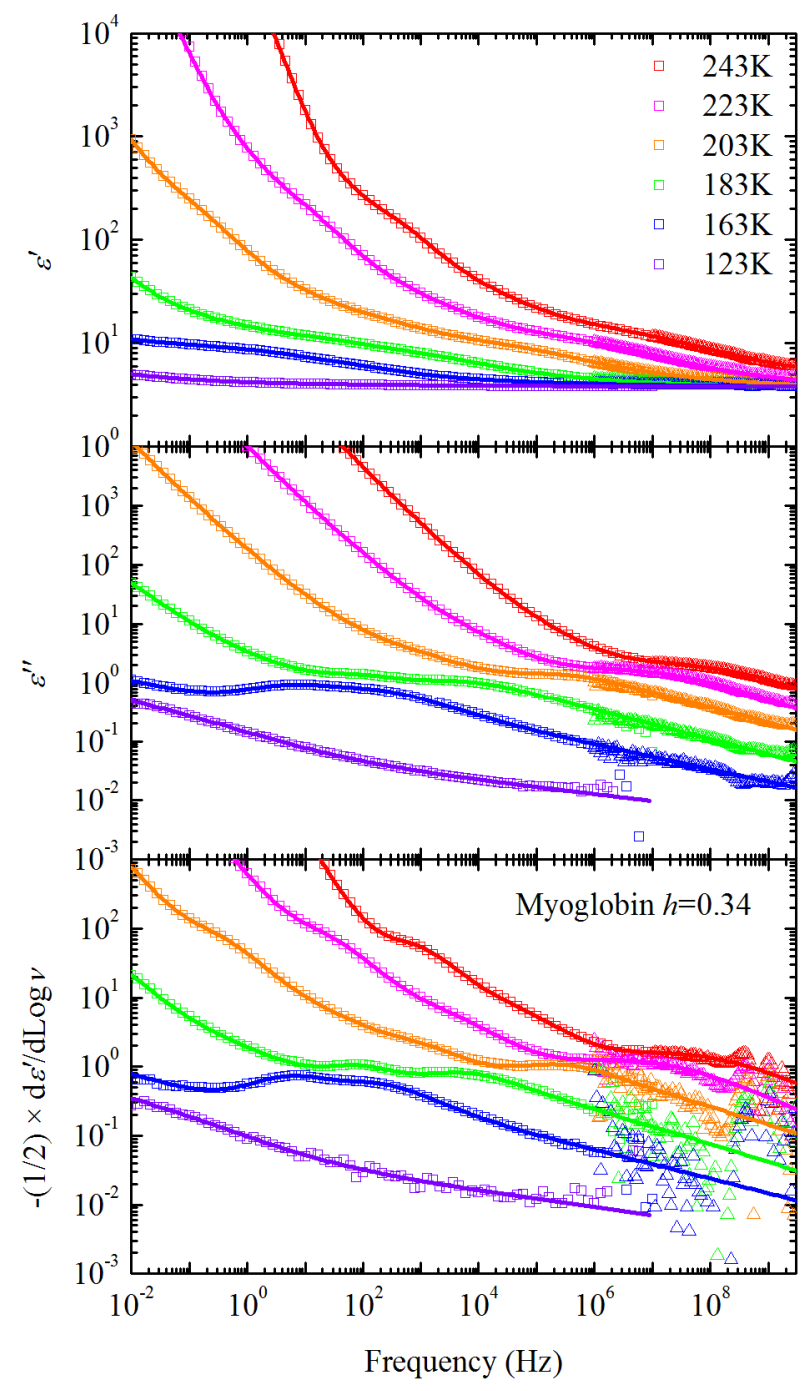

Fig. 2: Complex permittivity spectra, real part (top panel), imaginary part (middle panel), and derivative spectra (bottom panel), of myoglobin at $h=0.34$ at representative temperatures. Symbols show experimental data, and solid lines show fits composed of dc-conductivity, electrode polarization, processes $\mathrm{B}, \mathrm{D}$, and $\mathrm{G}$ (and $\mathrm{E}$ and $\mathrm{F}$ at $T \leq 203 \mathrm{~K}$ ).

At $T \geq 180 \mathrm{~K}$ the complex permittivity spectra can be fit with the superposition of three Cole-Cole processes [42] (processes B, D, and G), a Cole-Davidson function [43] for the electrode polarization (EP), and a dc-conductivity (Fig.3): 


$$
\varepsilon^{*}(\omega)=\varepsilon_{\infty}+\sum_{i=B, D, G} \frac{\Delta \varepsilon_{i}}{\left[1+\left(i \omega \tau_{i}\right)^{\beta_{i}}\right]}+\frac{\Delta \varepsilon_{E P}}{\left[1+\left(i \omega \tau_{E P}\right)\right]^{\gamma E P}}+\frac{\sigma_{0}}{i \omega \varepsilon_{0}}
$$

Here $\Delta \varepsilon_{i}, \tau_{i}, \beta_{i}$ are the dielectric relaxation amplitude, the relaxation time and stretching of the $i$ process, respectively; $\varepsilon_{\infty}$ is the limiting value of dielectric constant at high frequency; $\sigma_{0}$ is dcconductivity; $\varepsilon_{0}$ is a vacuum permittivity. The Cole-Cole function instead of e.g. HavriliakNegami function [44] was chosen to reduce the numbers of free fit parameters. This choice, however, does not affect the estimates of the characteristic relaxation times (defined using the frequency of the peak maximum) and their temperature dependences that are the main focus of our studies.

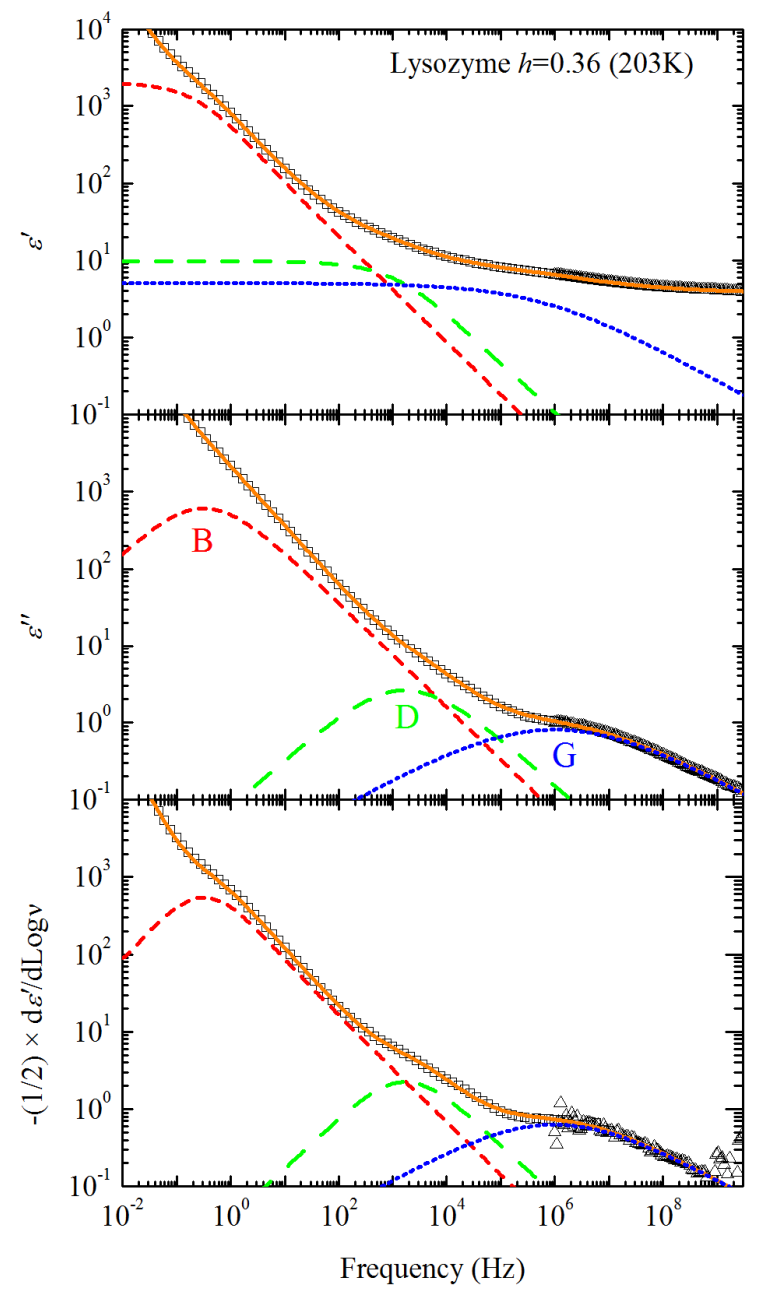


Fig. 3: Detail of curve fitting for complex permittivity spectra of lysozyme at $h=0.36$ and $T=$ 203 K. Dashed lines with three different intervals correspond to process B, D, and G as being specified in the middle panel. Electrode polarization is at much lower frequency, and only its high frequency tail can be recognized at this temperature. In the imaginary part, dc conductivity is also superimposed (is not shown). Solid lines show total fit to the Eq. 2 with all these components.

Fits at $T<180 \mathrm{~K}$ cannot be performed without unambiguity. The process marked $\mathrm{G}$ at higher temperatures becomes a double peak with an extended high-frequency tail. The latter has been detected in many glass-forming systems and is usually called the NCL (or excess wing) [45-51]. Figure 4 presents an example of the fit in this temperature range with five processes, but it is not a unique scenario. It is not obvious which of the two peaks in the double peak is the same G peak that was observed at higher $T$, and whether these might be two new peaks that overlap with the $G$ peak. For simplicity we assume that one of the bimodal peaks ( $E$ or $F$ ) is the same G peak, and G'-peak is used to fit the NCL. Since the NCL does not really has a separate peak, the process G' maximum was placed at the midpoint between the processes $\mathrm{E}$ and $\mathrm{F}$ to simplify the fit. 


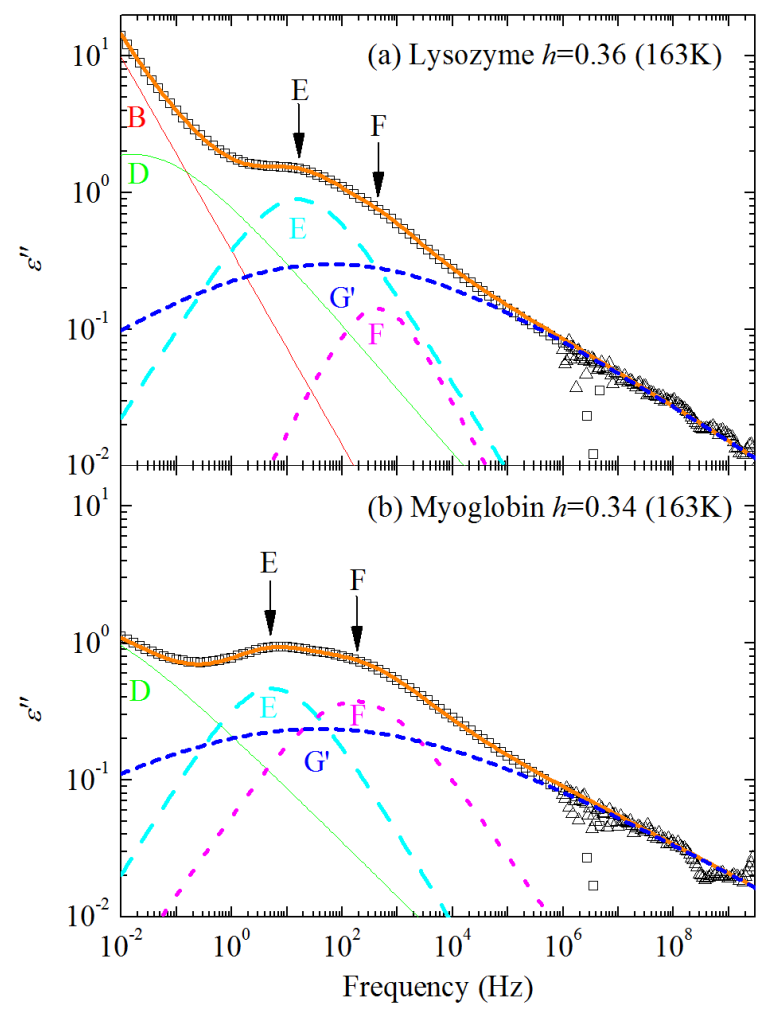

Fig. 4: Detail of curve fitting for complex permittivity spectra of lysozyme at $h=0.36$ and $T=$ $163 \mathrm{~K}$, where bimodal peak appears. The dashed lines present processes E, F and G', while the solid lines present processes B and D. The solid lines through the experimental data (symbols) present total fit curves. Peaks of processes E and F are also shown by arrows. Since process G' represents the NCL, its peak was just placed at the middle of the double peak.

\section{Results and Discussion}

Nearly Constant Loss

Analysis of the spectra (Figs. 1 - 4) suggests the existence of at least 3 well resolved dielectric relaxation peaks at $T>180 \mathrm{~K}$ and appearance of one more peak at lower $T$. Let's start 
our discussion with higher frequency data. It has been suggested in a few papers (e.g. [8, 9, 52, 53]) that there is a high frequency peak in the $\mathrm{MHz}$ range with very weak temperature dependence. As displayed in the magnified high frequency spectra (Fig. 5), our detailed analysis with accurate measurements extended to higher frequencies clearly exclude the existence of the high frequency peak. As temperature is decreased to below $180 \mathrm{~K}$, it shows strong NCL that is a usual high frequency tail of structural relaxation observed in many glass-forming systems. In particular, it has been observed in $\mathrm{LiCl}-\mathrm{H}_{2} \mathrm{O}$ system [51] and can be a part of the hydration water relaxation process that we marked $G$ in our fits. We suspect that the earlier studies $[8,52]$ misinterpreted spectral fluctuations and uncertainty on the edge of the low-frequency spectrometer (in the $\mathrm{MHz}$ range) as an additional relaxation peak. Our analysis reveals small values of the exponent for the high-frequency tail that decreases with temperature decrease, $\varepsilon " \propto$ $v^{-0.24}$ at $163 \mathrm{~K}$ and $\propto v^{-0.17}$ at $143 \mathrm{~K}$ (Fig. 5). This behavior is usual for NCL. Thus our accurate measurements in the $\mathrm{MHz}-\mathrm{GHz}$ range at lower $T$ clearly demonstrate the existence of the NCL only. This result is consistent with the recent measurements on bovine serum albumin (BSA) [17] that also found only NCL in this frequency range at lower $T$. Thus the NCL is a general feature in the dielectric relaxation spectra of various hydrated protein powders. 


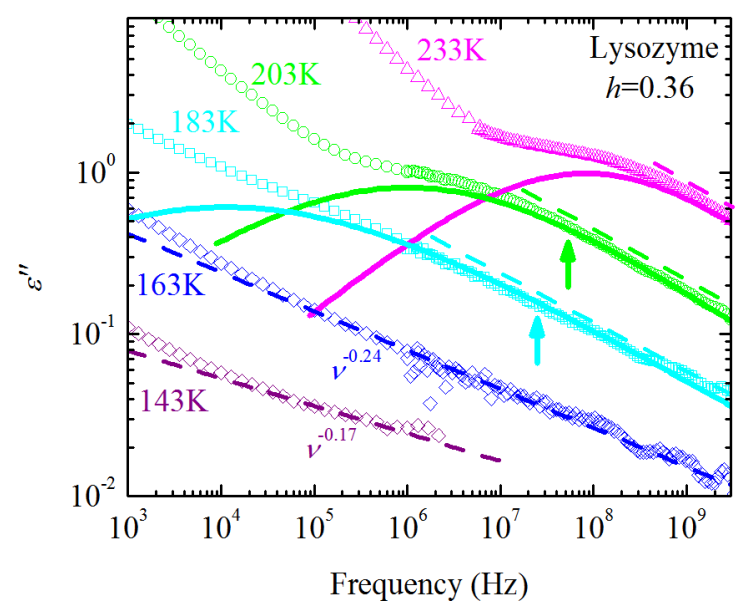

Fig. 5: The high frequency part of the dielectric spectra of lysozyme at $h=0.36$. Full lines show process $\mathrm{G}$, while dashed lines indicate power law behavior $v^{-0.24}$ at $163 \mathrm{~K}$ and $v^{-0.17}$ at $143 \mathrm{~K}$. Arrows represent the reported in [8] peak positions of the fast process. Our detailed analysis reveals no peak, just an NCL.

\section{Main Process}

The only process that is well resolved in the dielectric spectra at $T>180 \mathrm{~K}$ is the process marked $\mathrm{G}$ in our fits (Figs. $1-3$ ). Because it is the only well-resolved peak, it was named the Main process in $[8,9]$, and we will use this name in the following discussion. There are three characteristic features of this process: (i) The relaxation time of this process has slightly superArrhenius temperature dependence down to $T \sim 200-180 \mathrm{~K}$ (Fig. 6); (ii) At $T \sim 170-190 \mathrm{~K}$ the temperature dependence of this process crosses over to an Arrhenius behavior with characteristic activation energy barrier $\sim 30-41 \mathrm{~kJ} / \mathrm{mol}$; (iii) It has strongly stretched rather symmetric spectral shape with the stretching exponent of the Cole-Cole function, $\beta_{\text {main }} \sim 0.4-0.5$ even at $T$ 200 K. Clear emergence of the NCL at lower temperatures complicates the analysis of spectral shape of the Main process at $T<180 \mathrm{~K}$. These characteristic features of the Main process have 
been reported earlier for lysozyme [8,9], myoglobin [52] and BSA [16, 17], and are general features of the dielectric spectra of protein powders. Moreover, as it has been discussed in several papers [54-56], it is the same process that has been observed in many other watercontaining samples and is often called $v$-process of water. In agreement with the earlier reports on the v-process [54-56], an unusual temperature dependence of the dielectric relaxation amplitude of the Main process can be recognized in Fig. 7 (i.e. $\Delta \varepsilon_{G}$ decreases with decreasing temperature). Because of the complexity of the spectrum below $180 \mathrm{~K}$ detailed $h$ dependence of the crossover temperature cannot be determined unambiguously in our study. However, a decrease of this crossover temperature with increase in hydration has been reported in [57].

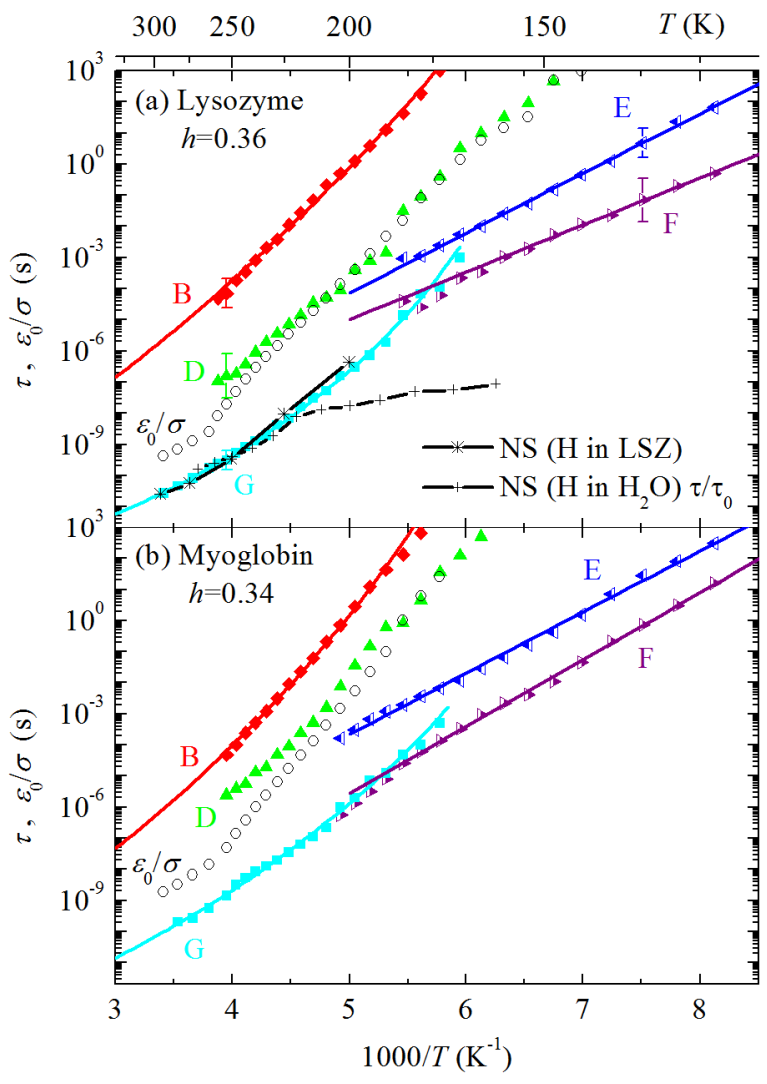


Fig. 6: Temperature dependence of the relaxation times of hydrated (a) lysozyme at $h=0.36$ and (b) myoglobin at $h=0.34$. The filled and half-filled symbols stand for dielectric relaxation times

of processes B, D, G, E and F. The open circles represent reciprocal conductivity via plateau value of the real part of complex conductivity. The star-symbols present neutron scattering relaxation times for non-exchangeable protons in protein molecules [8], whereas plus-symbols represent neutron scattering relaxation times for protons in hydration water [7].

The dielectric relaxation spectra are not sufficient to unravel the microscopic details of the molecular motion involved in the Main process and results of other techniques should be involved. Using hydrogen/deuterium contrast neutron scattering can separate contribution of hydration water and of the protein to the relaxation spectra $[58,59]$. Detailed analysis of neutron scattering spectra of lysozyme (with important correction for methyl group contribution) revealed that both hydration water and protein relaxation has similar relaxation time and its temperature dependence [8, 9] (Fig.6a). Moreover, the stretching of the protein relaxation process in the neutron scattering spectra of lysozyme and myoglobin is also very strong $[1,8,9]$. NMR studies also revealed relaxation of many side groups in the same $10-100 \mathrm{ps}$ time scale at ambient conditions $[3,4]$. All these results clearly suggest that the Main process is a coupled protein-hydration water relaxation [9]. It involves rotation and translation motion of water coupled to localized fluctuations of protein. According to detailed analysis of neutron scattering and MD simulations results atomic motions in this protein relaxation process are localized to distances $~ 1-3 \AA[10,60-62]$.

Extrapolation of the data for this process in different proteins to room temperature provides estimates of the characteristic relaxation time $\sim 10-30$ ps (see e.g. Fig. 6 and Ref. [12]). Studies of many proteins in dilute solutions at ambient temperature also revealed the 
process around the same relaxation time $[5,6,13,15,18,19]$. In the case of dilute solutions it is usually ascribed to hydration water. Thus the Main process exists in hydrated protein powders and solutions and is a coupled protein-hydration water relaxation process. It involves rotational and translational water motions and localized fluctuations of the protein [9, 11, 12, 62]. According to $[8,63,64]$, it is the process that causes the sharp rise of the mean-squared atomic displacements (the so-called dynamic transition) at above $\sim 220 \mathrm{~K}$ in hydrated proteins measured by neutron scattering.

\section{Slow Process}

At frequencies below the Main peak, ionic conductivity dominates the dielectric loss spectra (Figs. 1 - 2). Several authors used insulating layers (e.g. a Teflon film) to suppress the ionic conductivity $[52,65,66]$. It is well-known that this layer leads to some artificial peaks in the dielectric relaxation spectra and their misinterpretations $[39,40]$. Thus we will not discuss any of the papers that used the insulating layers in the dielectric measurements. Detailed analysis of the dielectric spectra (Fig. 3) clearly revealed two more processes (B and D) at frequencies below the Main process. The process D was called Slow process in $[9,12]$, and we will use the same name here. This process appears in the frequency range where conductivity relaxation mode and MW polarization effects in heterogeneous systems are expected $[39,40]$.

Analysis of the characteristic relaxation time of the Slow (D) process indeed shows that its temperature variations follow the conductivity contribution and even quantitatively $\tau_{\text {slow }}$ is close to the conductivity relaxation time $\sim \mathcal{E}_{0} / \sigma$ (Fig. 6). This does not necessarily mean that the Slow process results from some interfacial effects (e.g. MW polarizations). It is also possible that the temperature dependence of the Slow process is controlled by the same mechanism that 
controls conductivity. To sort out possible effects of the interfaces and their polarization we performed additional experiments. The MW polarization can appear in the spectra of protein powders due to several reasons, including air gaps between powder sample and electrodes and some voids in the sample. The former should depend on the contact condition at the surface of the sample, such as area of air gaps, and their thicknesses. Voids in the samples are expected to depend on the packing fraction of powder. Therefore, we examined a variety of contact conditions to exclude the possibility for the former, and a variety of packing fraction in order to see the effect from the latter. Several conductive materials, such as gold leafs, silver powder (2 $3.5 \mu \mathrm{m}$ of diameter) and indium foils (125 $\mu \mathrm{m}$ of thickness), were inserted between both sides of pellet and electrodes. These materials provide significantly different surface conditions as they change their shape according to the pellet surface roughness. On the other hand, powders at a variety of packing fractions were also examined: (i) pellet made by compressing powder via press applying about $150 \mathrm{MPa}$ of pressure, (ii) tightly packed powder prepared by pressing powder by hand at pressure less than $0.4 \mathrm{MPa}$, (iii) loosely packed powder made by just sandwiching powder by electrodes with tiny pieces of spacers. It was confirmed that the last one was certainly loosely packed from the view point of dielectric spectrum. In its dielectric spectrum, clear MW process showed up additionally at frequency between the process B and D, and thereby relaxation intensity of the process B was decreased considerably while the process D did not exhibit significant change. We confirm that this modification of the dielectric spectrum can be reproduced based on the spectrum of tightly packed powder (sample 1), assuming equivalent circuit composed of the sample 1 and air gap partially blocking dc-conductivity. Thus these analyses suggest that neither processes B nor D are attributed to insufficient packing, i.e. 
the MW effect from voids or contact effects, and present relaxation processes in hydrated proteins.

Another complication with analysis of this process is the contribution of ice relaxation in the same frequency range $[17,67]$. Excess water (above a particular hydration level that depends on protein) would usually crystallize at lower temperatures. The ice formed in this process will also have dielectric relaxation modes that will be in the same frequency range as the Slow process, $10^{-4}-10^{-2} \mathrm{~s}$ at around $200 \mathrm{~K}$. However, the Slow process at this temperature has significantly larger activation energy, $\sim 70 \mathrm{~kJ} / \mathrm{mol}$, compared to ice Ih relaxation, $\sim 19 \mathrm{~kJ} / \mathrm{mol}$ $(250-170 \mathrm{~K})$ [67]. Moreover, in general ice contribution would have significant difference on initial cooling and heating that provides a way to differentiate it from other dielectric relaxations. Therefore, the contribution from ice could be distinguished from the Slow process.

In the following analysis we only use the data on pressed pellets with good electrical contact that reduces the MW polarization contribution. We also will use samples with $h<0.4$ where only small formation of ice is expected. Characteristic relaxation time of the Slow process appears $\sim 3$ orders slower than $\tau_{\text {main }}$ of the Main process and also shows slightly non-Arrhenius temperature dependence at $T>200 \mathrm{~K}$ (Fig. 6). Its amplitude is comparable and even a bit higher than the amplitude of the Main process (Fig. 7). This process has been detected in many earlier studies of protein powders $[9,12,16]$. Moreover, extrapolation of its relaxation time to room temperature provides $\tau_{\text {slow }} \sim 10 \mathrm{~ns}$ (Fig. 6), which is close to the relaxation time of the dielectric process observed in dilute protein solutions at frequencies above tumbling $[15,18,19]$. 


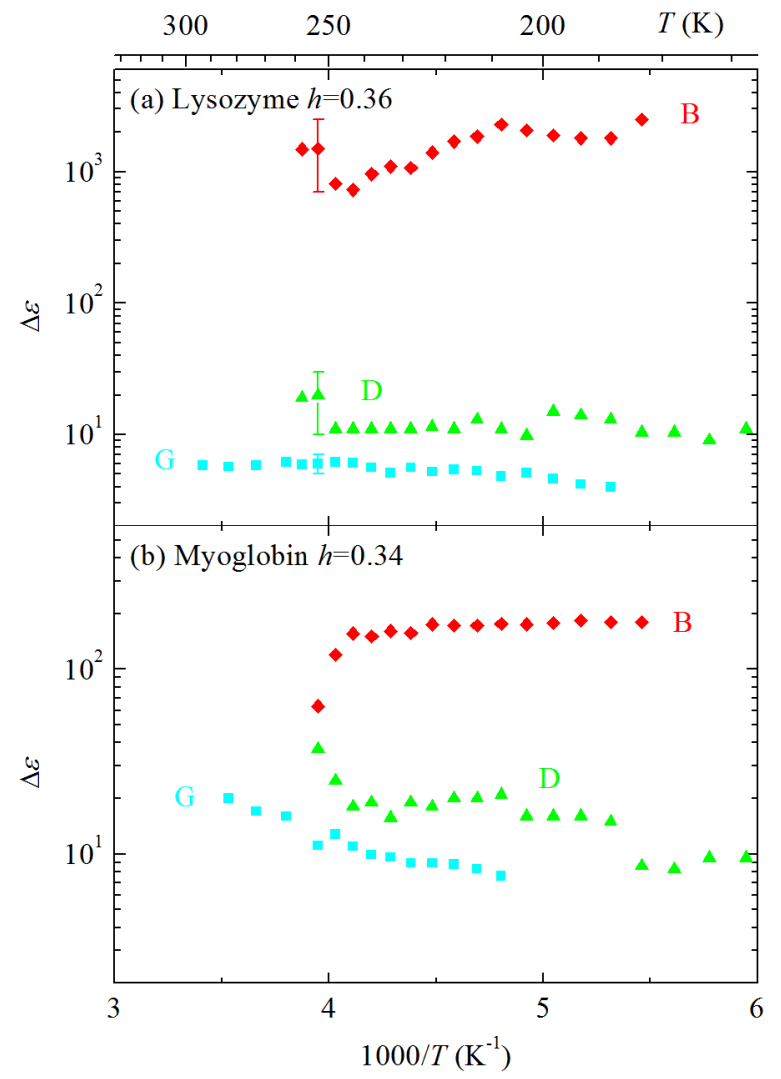

Fig. 7: Temperature dependence of the dielectric amplitudes for processes in hydrated (a) lysozyme at $h=0.36$ and (b) myoglobin at $h=0.34$. Because of the appearance of the double peak, amplitudes at $T \leq 183 \mathrm{~K}$ for lysozyme and $T \leq 203 \mathrm{~K}$ for myoglobin were not analyzed.

Once again, analysis of the dielectric relaxation spectra only does not provide information on microscopic origin of the Slow process. In many papers this process is ascribed to hydration water that is tightly bound to protein surface $[15,18,20,22]$. In that case, however, it is assumed that the hydration water can be $\sim 10^{3}$ times slower than the bulk water. Moreover, the amplitude of this process suggests that about half of the hydration water should be tightly bounded to the protein surface. Analysis of neutron scattering, MD simulations [10, 13] and NMR data $[5,6]$ suggests that major part of protein's hydration water is slowed down only by a 
factor $2-4$ (consistent with the data for the Main process), and more than $90 \%$ of hydration water molecules are exchanged with the bulk water on time scale faster than $1 \mathrm{~ns}$ [12]. This excludes, in our view, a possibility to ascribe the Slow process to tightly bounded hydration water.

Neutron spin-echo measurements on protein solutions clearly identified process in $5-30$ ns time range of several proteins and ascribe this process to domain motions [68-72]. Combined analysis of MD simulations and neutron scattering data for hydrated protein powders also revealed a ns-relaxation process in proteins that was ascribed to some conformational jumps $[10$, 11]. NMR studies also found several backbone and side group motions in proteins on the ns time scale $[3,4]$. Although clear microscopic picture of the Slow process is still absent, it seems obvious that this is the relaxation of protein that involves large scale motions, including domain motions and fluctuations of secondary structures. We want to note that the appearance of the Slow process in the dielectric relaxation spectra might be visible due to motion of the water continuum caused by the protein motions. But it seems obvious to us that this relaxation process is intrinsic to proteins and not to the tightly bounded hydration water.

The Process with Anomalously Large Intensity

The dielectric spectra, especially the derivative spectra, clearly show the existence of a process at frequencies below the Slow process (process B in Figs. $1-3$ ). This process has Anomalously large Intensity ( $\Delta \varepsilon_{\mathrm{AI}} \sim 10^{3}$ in lysozyme and $\Delta \varepsilon_{\mathrm{AI}} \sim 200$ in myoglobin), and we will call it AI-process. It has slightly non-Arrhenius temperature dependence and relaxation time that depends strongly on hydration level (Fig. 8). Characteristic relaxation time of this process at hydration level $h>0.3$ is $\sim 10^{6}-10^{7}$ times slower than the Main process, and it reaches $\tau_{\mathrm{AI}} \sim 10^{2}$ 
$-10^{3} \mathrm{~s}$ at $T \sim 180 \mathrm{~K}$ (Figs. 6, 8). Similar process has been found in recent dielectric spectroscopy studies of BSA $[16,17]$. In this case the amplitude of the process was even higher, $\Delta \varepsilon_{\mathrm{AI}} \sim 10^{4}$. In all these cases, extrapolation of the relaxation time of this process to room temperature suggests that it should be $\sim 1-10 \mu$ s (Figs. 6,8 ).

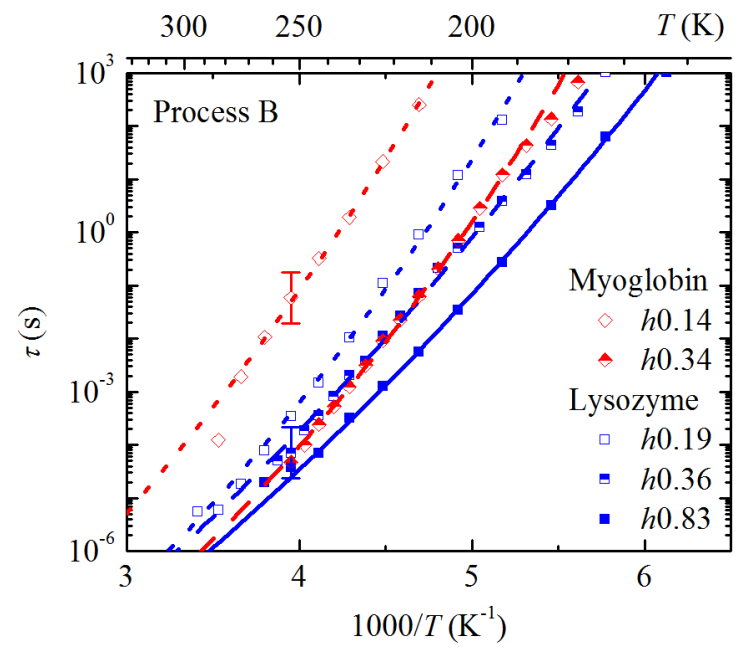

Fig. 8: Temperature dependence of relaxation times of the process B (AI-process) at various hydration levels.

The most interesting is a possible connection of this process to the glass transition in hydrated protein powders. This idea has been already discussed in $[16,17]$. The temperature at which structural relaxation time ( $\alpha$-process) in glass-forming systems reaches $10^{2}-10^{3} \mathrm{~s}$ is usually defined as $T_{\mathrm{g}}$. The characteristic relaxation time of the AI-process indeed reaches these values at temperatures where calorimetry show some weak signs of the glass transition $[16,17$, 73, 74]. Moreover, many studies of protein powders with different techniques suggested existence of a broad glass transition at $T \sim 160-200 \mathrm{~K}$ that depends on hydration level [74-78]. 
Analyzing temperature dependence of $\tau_{\mathrm{AI}}$ in lysozyme and myoglobin at various hydration level, we fit the data to Vogel-Fulcher-Tammann (VFT) equation:

$$
\tau_{A I}=\tau_{0} \exp \left[B /\left(T-T_{0}\right)\right]
$$

The results of the fit are presented in the Table 1 and help to estimate the expected $T_{\mathrm{g}}$ as a temperature at which $\tau_{\mathrm{AI}}$ reaches $100 \mathrm{~s}$. Comparison of so estimated $T_{\mathrm{g}}$ for lysozyme and myoglobin are presented in the Fig. 9 together with other estimates of $T_{\mathrm{g}}$ published in literature [57, 73, 75]. This comparison clearly suggests that the AI-process might be the structural relaxation of hydrated proteins. Moreover, as has been suggested in earlier papers [53, 54, 56, $57,79]$, the crossover in temperature dependence of the Main (or $v$-) process at $T \sim 170-200 \mathrm{~K}$ (Fig. 6) might be also related to crossing the $T_{\mathrm{g}}$ of the system. Fragility index $m$, defined as $m=\left[d \log \tau / d\left(T_{g} / T\right)\right]_{T=T_{g}}[80]$, was calculated from the obtained parameters of VFT equation, and is presented in the Table 1. Assuming the AI-process reflects structural relaxation, our analysis suggests that the hydrated protein systems are rather "strong", consistent with the calorimetric study by Green and Angell [81]. Such a low fragility is typical for network glassformers such as silica [80] and water [82].

Table 1: Parameters for Vogel-Fulcher-Tammann (VFT) equation obtained from this study. A symbol $h$ indicates hydration level. Symbols $B$ and $T_{0}$ are parameters for the VFT equation. Glass transition temperature $T_{\mathrm{g}}$ is defined as the temperature where relaxation time reaches 100 s. Fragility index $m[80]$ and activation energy $\Delta H$ at $T_{\mathrm{g}}$ are also listed.

\begin{tabular}{|c|c|c|c|c|c|}
\hline$h$ & $\begin{array}{l}B \\
\mathrm{~K}\end{array}$ & $\begin{array}{l}T_{0} \\
\mathrm{~K}\end{array}$ & $\begin{array}{l}T_{\mathrm{g}} \\
\mathrm{K}\end{array}$ & $m$ & $\begin{array}{c}\Delta H\left(T_{g}\right) \\
\mathrm{kJ} \cdot \mathrm{mol}^{-1}\end{array}$ \\
\hline \multicolumn{6}{|l|}{ Lysozyme } \\
\hline 0.83 & 4282 & 55.13 & 171.4 & 23.6 & 77.4 \\
\hline 0.36 & 4515 & 58.96 & 181.5 & 23.7 & 82.3 \\
\hline 0.19 & 4180 & 81.89 & 195.3 & 27.5 & 103.0 \\
\hline
\end{tabular}




\begin{tabular}{rrrrrr}
\multicolumn{1}{c|}{ Myoglobin } & & & & & \\
\cline { 1 - 3 } 0.34 & 3863 & 81.80 & 186.6 & 28.5 & 101.8 \\
0.14 & 5135 & 77.43 & 216.8 & 24.9 & 103.3 \\
\hline \hline
\end{tabular}

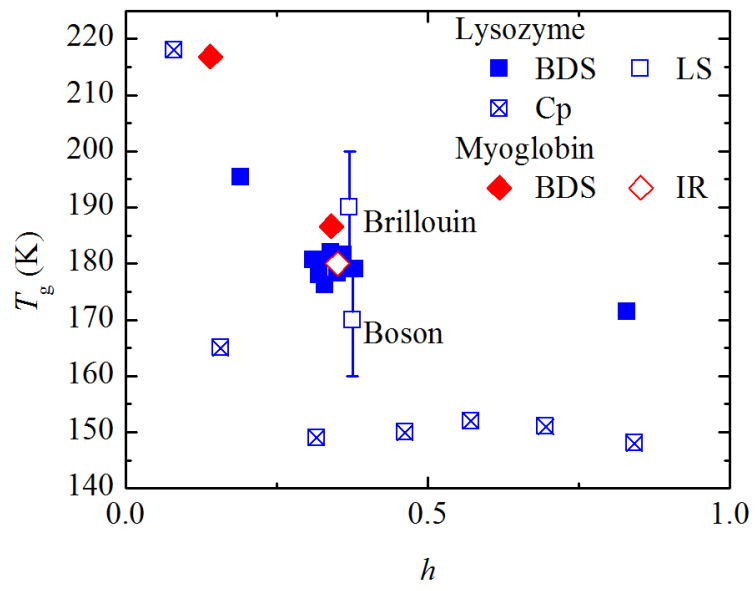

Fig. 9: Hydration level dependence of the glass transition temperature. Solid symbols show $T_{\mathrm{g}}$ obtained in this work, defined as temperature where the relaxation time of the process B reaches $100 \mathrm{~s}$. Open and crossed symbols indicates representative literature data of $T_{\mathrm{g}}$ : LS (open square) indicates inflection of the temperature dependence of the Brillouin peak and the Boson peak of lysozyme measured by light scattering spectroscopy [57]; IR (open diamond) shows inflection of temperature dependence of OD stretching vibrational frequency of myoglobin [75]; Cp (crossed square) indicates onset temperature of the broad heat capacity jump of hydrated crystalline lysozyme [73].

A possible connection of the AI-process to the glass transition in hydrated proteins makes especially intriguing to understand the microscopic nature of this process. Unfortunately, the time-scale of this process does not allow to study it with neutron scattering, because the latter has limitations at a few hundreds of ns time scale. The current MD simulations are just reaching this time and we are aware of only one paper that indeed found a protein relaxation in the $\mu$ s time scale [83]. It is especially challenging for MD simulations because equilibration of the system should be at least 10 times longer than the characteristic time of the process we intend to study. 
This means that simulations should be performed on time scales extending to $\sim 100 \mu \mathrm{s}$, which is not easily feasible at the current stage. We are also not aware of any NMR studies of protein dynamics at these time scales. Moreover, the dielectric studies of dilute protein solutions are not able to detect this process because it appears on the time scale significantly longer than the tumbling time $(\sim 10-100 \mathrm{~ns}[15,18-20,22])$.

The anomalously large amplitude of this process suggests that it should be related to a strong polarization of the protein or its surface, to motion of charges and counter-ions. A strong increase of the amplitude of this process by going from myoglobin $(\Delta \varepsilon \sim 200)$ to lysozyme $(\Delta \varepsilon \sim$ $\left.10^{3}\right)$, and to BSA $\left(\Delta \varepsilon \sim 10^{4}\right)$ provides some additional hint to the microscopic nature of this process and its anomalously large intensity in the dielectric spectra. Recently Matyushov and coworkers have suggested a ferroelectric hydration shell around protein [84]. This kind of cooperative polarization might be responsible for such an intense dielectric process. It would be interesting if this model can explain the amplitude of AI-process and its difference between myoglobin, lysozyme and BSA. It would be also important to extend MD simulations to the $\mu$ s time scale and try to identify this process and its microscopic nature through simulations. If this process is indeed responsible for the glass transition in proteins, understanding of its microscopic mechanism maybe crucial for understanding of many biophysical and biochemical processes in biological macromolecules.

\section{Conclusions}

Detailed analysis of the dielectric spectra of hydrated lysozyme and myoglobin revealed three major relaxation modes at $T>180 \mathrm{~K}$. This result is consistent with the recent dielectric studies on BSA [16, 17], if we exclude ice relaxations also detected in these studies. Our analysis 
excludes existence of the fast relaxation process suggested in $[8,9,52,53]$, and ascribes these earlier observations to signal fluctuations and accuracy of the measurements on the edge of the spectrometers in the MHz frequency range. Instead of this process we observed an NCL (as the high-frequency tail of the Main process), consistent with the observation of the near constant loss in BSA [17].

The observed three major processes are: (i) Main process with characteristic $\tau_{\text {main }} \sim 10-50$ ps at room $T$; (ii) Slow process with $\tau_{\text {slow }} \sim 5-30$ ns at room $T$; (iii) Anomalously Intense process with $\tau_{\mathrm{AI}} \sim 1-10 \mu \mathrm{s}$ at room $T$ (Fig. 6). Our analysis and earlier literature data suggest that the Main process is a coupled protein-hydration water relaxation, where hydration water exhibits rotational and translational motions, while protein has only localized conformational fluctuations with characteristic amplitude $\sim 1-3 \AA$ [10, 60-62]. The microscopic nature of the Slow process is less understood. In our view, we can exclude the contribution of tightly bounded hydration water to this process. Neutron scattering, MD simulations and NMR studies all suggest that this is a protein relaxation process, most probably large scale domain-like motions [68-72]. Even less is understood about the AI-process. It appears on the time scale not easily accessible for current neutron scattering and MD-simulations. However, a possible connection of the AIprocess to the glass transition in hydrated proteins makes it especially intriguing and important to understand the microscopic nature of this process. We expect that MD simulations extended to the microsecond time range will provide microscopic details of this relaxation process.

Acknowledgments: We thank Dr. Feldman for helpful discussions, MN acknowledges UTBattelle financial support during his stay in the US. APS acknowledges DOE EPSCoR program. 


\section{References}

[1] W. Doster, S. Cusack, W. Petry, Dynamical transition of myoglobin revealed by inelastic neutron scattering, Nature, 337 (1989) 754-756.

[2] K. Henzler-Wildman, D. Kern, Dynamic personalities of proteins, Nature, 450 (2007) 964-972.

[3] M. Buck, J. Boyd, C. Redfield, D.A. MacKenzie, D.J. Jeenes, D.B. Archer, C.M. Dobson, Structural Determinants of Protein Dynamics: Analysis of ${ }^{15} \mathrm{~N}$ NMR Relaxation Measurements for Main-Chain and Side-Chain Nuclei of Hen Egg White Lysozyme, Biochemistry, 34 (1995) 4041-4055.

[4] A. Krushelnitsky, D. Reichert, Solid-state NMR and protein dynamics, Prog. Nucl. Magn. Reson. Spectrosc., 47 (2005) 1-25.

[5] C. Mattea, J. Qvist, B. Halle, Dynamics at the protein-water interface from ${ }^{17} \mathrm{O}$ spin relaxation in deeply supercooled solutions, Biophys. J., 95 (2008) 2951-2963.

[6] K. Modig, E. Liepinsh, G. Otting, B. Halle, Dynamics of protein and peptide hydration, J. Am. Chem. Soc., 126 (2004) 102-114.

[7] S.H. Chen, L. Liu, E. Fratini, P. Baglioni, A. Faraone, E. Mamontov, Observation of fragile-to-strong dynamic crossover in protein hydration water, Proc. Natl. Acad. Sci. U.S.A., 103 (2006) 9012-9016.

[8] S. Khodadadi, S. Pawlus, J.H. Roh, V.G. Sakai, E. Mamontov, A.P. Sokolov, The origin of the dynamic transition in proteins, J. Chem. Phys., 128 (2008) 195106.

[9] S. Khodadadi, S. Pawlus, A.P. Sokolov, Influence of Hydration on Protein Dynamics: Combining Dielectric and Neutron Scattering Spectroscopy Data, J. Phys. Chem. B, 112 (2008) 14273-14280.

[10] L. Hong, N. Smolin, B. Lindner, A.P. Sokolov, J.C. Smith, Three Classes of Motion in the Dynamic Neutron-Scattering Susceptibility of a Globular Protein, Phys. Rev. Lett., 107 (2011) 148102.

[11] L. Hong, X. Cheng, D.C. Glass, J.C. Smith, Surface Hydration Amplifies Single-Well Protein Atom Diffusion Propagating into the Macromolecular Core, Phys. Rev. Lett., 108 (2012) 238102.

[12] S. Khodadadi, J.E. Curtis, A.P. Sokolov, Nanosecond Relaxation Dynamics of Hydrated Proteins: Water versus Protein Contributions, J. Phys. Chem. B, 115 (2011) 6222-6226.

[13] F. Sterpone, G. Stirnemann, D. Laage, Magnitude and Molecular Origin of Water Slowdown Next to a Protein, J. Am. Chem. Soc., 134 (2012) 4116-4119.

[14] F. Kremer, A. Schönhals, Broadband Dielectric Spectroscopy, Springer, Berlin/Heidelberg/New York, 2002.

[15] C. Cametti, S. Marchetti, C.M.C. Gambi, G. Onori, Dielectric Relaxation Spectroscopy of Lysozyme Aqueous Solutions: Analysis of the $\delta$-Dispersion and the Contribution of the Hydration Water, J. Phys. Chem. B, 115 (2011) 7144-7153.

[16] A. Panagopoulou, A. Kyritsis, N. Shinyashiki, P. Pissis, Protein and Water Dynamics in Bovine Serum Albumin-Water Mixtures over Wide Ranges of Composition, J. Phys. Chem. B, 116 (2012) 45934602.

[17] N. Shinyashiki, W. Yamamoto, A. Yokoyama, T. Yoshinari, S. Yagihara, R. Kita, K.L. Ngai, S. Capaccioli, Glass Transitions in Aqueous Solutions of Protein (Bovine Serum Albumin), J. Phys. Chem. B, 113 (2009) 14448-14456.

[18] N. Miura, N. Asaka, N. Shinyashiki, S. Mashimo, Microwave Dielectric Study on Bound Water of Globule Proteins in Aqueous-Solution, Biopolymers, 34 (1994) 357-364.

[19] A. Oleinikova, P. Sasisanker, H. Weingärtner, What can really be learned from dielectric spectroscopy of protein solutions? A case study of ribonuclease A, J. Phys. Chem. B, 108 (2004) 84678474. 
[20] M. Wolf, R. Gulich, P. Lunkenheimer, A. Loidl, Relaxation dynamics of a protein solution investigated by dielectric spectroscopy, Biochim. Biophys. Acta, 1824 (2012) 723-730.

[21] K. Yokoyama, T. Kamei, H. Minami, M. Suzuki, Hydration study of globular proteins by microwave dielectric spectroscopy, J. Phys. Chem. B, 105 (2001) 12622-12627.

[22] R. Pethig, Protein-Water Interactions Determined by Dielectric Methods, Annu. Rev. Phys. Chem., 43 (1992) 177-205.

[23] J.F. Brandts, R.J. Oliveira, C. Westort, Thermodynamics of protein denaturation. Effect of pressu on the denaturation of ribonuclease A, Biochemistry, 9 (1970) 1038-1047.

[24] S.A. Hawley, Reversible pressure--temperature denaturation of chymotrypsinogen, Biochemistry, 10 (1971) 2436-2442.

[25] Y.O. Kamatari, H. Yamada, K. Akasaka, J.A. Jones, C.M. Dobson, L.J. Smith, Response of native and denatured hen lysozyme to high pressure studied by ${ }^{15} \mathrm{~N} /{ }^{1} \mathrm{H}$ NMR spectroscopy, Eur. J. Biochem., 268 (2001) 1782-1793.

[26] A. Zipp, W. Kauzmann, Pressure denaturation of metmyoglobin, Biochemistry, 12 (1973) 42174228.

[27] B.W. Matthews, Solvent content of protein crystals, J. Mol. Biol., 33 (1968) 491-497.

[28] I.D. Kuntz, Hydration of macromolecules. III. Hydration of polypeptides, J. Am. Chem. Soc., 93 (1971) 514-516.

[29] F.M. Richards, The interpretation of protein structures: Total volume, group volume distributions and packing density, J. Mol. Biol., 82 (1974) 1-14.

[30] K. Gekko, H. Noguchi, Compressibility of globular proteins in water at 25.degree.C, J. Phys. Chem., 83 (1979) 2706-2714.

[31] K. Gekko, Y. Hasegawa, Compressibility-structure relationship of globular proteins, Biochemistry, 25 (1986) 6563-6571.

[32] Y.-Z. Wei, A.C. Kumbharkhane, M. Sadeghi, J.T. Sage, W.D. Tian, P.M. Champion, S. Sridhar, M.J. McDonald, Protein Hydration Investigations with High-Frequency Dielectric Spectroscopy, J. Phys. Chem., 98 (1994) 6644-6651.

[33] E. Mylonas, D.I. Svergun, Accuracy of molecular mass determination of proteins in solution by small-angle X-ray scattering, J. Appl. Crystallogr., 40 (2007) s245-s249.

[34] A. Donev, I. Cisse, D. Sachs, E.A. Variano, F.H. Stillinger, R. Connelly, S. Torquato, P.M. Chaikin, Improving the Density of Jammed Disordered Packings Using Ellipsoids, Science, 303 (2004) 990-993.

[35] H.M. Jaeger, S.R. Nagel, Physics of the Granular State, Science, 255 (1992) 1523-1531.

[36] J.C. Kendrew, G. Bodo, H.M. Dintzis, R.G. Parrish, H. Wyckoff, D.C. Phillips, A three-dimensional model of the myoglobin molecule obtained by x-ray analysis, Nature, 181 (1958) 662-666.

[37] C.C. Blake, D.F. Koenig, G.A. Mair, A.C. North, D.C. Phillips, V.R. Sarma, Structure of hen eggwhite lysozyme. A three-dimensional Fourier synthesis at 2 Angstrom resolution, Nature, 206 (1965) 757-761.

[38] K. Murata, A. Hanawa, R. Nozaki, Broadband complex permittivity measurement techniques of materials with thin configuration at microwave frequencies, J. Appl. Phys., 98 (2005) 084107.

[39] R. Richert, Insulated electrodes for eliminating conductivity in dielectric relaxation experiments, Eur. Phys. J. B, 68 (2009) 197-200.

[40] R. Richert, A. Agapov, A.P. Sokolov, Appearance of a Debye process at the conductivity relaxation frequency of a viscous liquid, J. Chem. Phys., 134 (2011) 104508.

[41] M. Wübbenhorst, J. van Turnhout, Analysis of complex dielectric spectra. I. One-dimensional derivative techniques and three-dimensional modelling, J. Non-Cryst. Solid., 305 (2002) 40-49.

[42] K.S. Cole, R.H. Cole, Dispersion and Absorption in Dielectrics I. Alternating Current Characteristics, J. Chem. Phys., 9 (1941) 341-351.

[43] D.W. Davidson, R.H. Cole, Dielectric Relaxation in Glycerol, Propylene Glycol, and n-Propanol, J. Chem. Phys., 19 (1951) 1484-1490.

[44] S. Havriliak, S. Negami, A complex plane representation of dielectric and mechanical relaxation processes in some polymers, Polymer, 8 (1967) 161-210. 
[45] A. Kudlik, S. Benkhof, T. Blochowicz, C. Tschirwitz, E. Rössler, The dielectric response of simple organic glass formers, J. Mol. Struct., 479 (1999) 201-218.

[46] J. Wiedersich, T. Blochowicz, S. Benkhof, A. Kudlik, N.V. Surovtsev, C. Tschirwitz, V.N. Novikov, E. Rössler, Fast and slow relaxation processes in glasses, J. Phys.: Condens. Matter, 11 (1999) A147A156.

[47] U. Schneider, R. Brand, P. Lunkenheimer, A. Loidl, Excess Wing in the Dielectric Loss of Glass Formers: A Johari-Goldstein $\beta$ Relaxation?, Phys. Rev. Lett., 84 (2000) 5560-5563.

[48] P. Lunkenheimer, R. Wehn, T. Riegger, A. Loidl, Excess wing in the dielectric loss of glass formers: further evidence for a $\beta$-relaxation, J. Non-Cryst. Solid., 307-310 (2002) 336-344.

[49] K.L. Ngai, An extended coupling model description of the evolution of dynamics with time in supercooled liquids and ionic conductors, J. Phys.: Condens. Matter, 15 (2003) S1107-S1125.

[50] C. Gainaru, R. Bohmer, R. Kahlau, E. Rossler, Energy landscape in molecular glasses probed by high-resolution dielectric experiments, Phys. Rev. B, 82 (2010) 104205.

[51] M. Nakanishi, P. Griffin, E. Mamontov, A.P. Sokolov, No fragile-to-strong crossover in $\mathrm{LiCl}^{-\mathrm{H}_{2} \mathrm{O}}$ solution, J. Chem. Phys., 136 (2012) 124512.

[52] H. Jansson, J. Swenson, The protein glass transition as measured by dielectric spectroscopy and differential scanning calorimetry, Biochim. Biophys. Acta, 1804 (2010) 20-26.

[53] J. Swenson, H. Jansson, J. Hedström, R. Bergman, Properties of hydration water and its role in protein dynamics, J. Phys.: Condens. Matter, 19 (2007) 205109.

[54] S. Cerveny, Á. Alegría, J. Colmenero, Universal features of water dynamics in solutions of hydrophilic polymers, biopolymers, and small glass-forming materials, Phys. Rev. E, 77 (2008) 031803.

[55] S. Capaccioli, K.L. Ngai, N. Shinyashiki, The Johari-Goldstein $\beta$-relaxation of water, J. Phys. Chem. B, 111 (2007) 8197-8209.

[56] K.L. Ngai, S. Capaccioli, S. Ancherbak, N. Shinyashiki, Resolving the ambiguity of the dynamics of water and clarifying its role in hydrated proteins, Philos. Mag., 91 (2011) 1809-1835.

[57] S. Khodadadi, A. Malkovskiy, A. Kisliuk, A.P. Sokolov, A broad glass transition in hydrated proteins, Biochim. Biophys. Acta, 1804 (2010) 15-19.

[58] J. Fitter, T. Gutberlet, J. Katsaras, Neutron Scattering in Biology, Springer, Berlin, 2006.

[59] J.S. Higgins, H.C. Benoît, Polymers and Neutron Scattering, Clarendon Press, Oxford, 1984.

[60] J.H. Roh, J.E. Curtis, S. Azzam, V.N. Novikov, I. Peral, Z. Chowdhuri, R.B. Gregory, A.P. Sokolov, Influence of hydration on the dynamics of lysozyme, Biophys. J., 91 (2006) 2573-2588.

[61] J.D. Nickels, V. García Sakai, A.P. Sokolov, Dynamics in Protein Powders on the NanosecondPicosecond Time Scale Are Dominated by Localized Motions, J. Phys. Chem. B, 117 (2013) 1154811555.

[62] J.D. Nickels, H. O'Neill, L. Hong, M. Tyagi, G. Ehlers, K.L. Weiss, Q. Zhang, Z. Yi, E. Mamontov, J.C. Smith, A.P. Sokolov, Dynamics of protein and its hydration water: neutron scattering studies on fully deuterated GFP, Biophys. J., 103 (2012) 1566-1575.

[63] W. Doster, The protein-solvent glass transition, Biochim. Biophys. Acta, 1804 (2010) 3-14.

[64] K.L. Ngai, S. Capaccioli, A. Paciaroni, Change of caged dynamics at Tg in hydrated proteins: Trend of mean squared displacements after correcting for the methyl-group rotation contribution, J. Chem. Phys., 138 (2013) 235102.

[65] F. Pizzitutti, F. Bruni, Glassy dynamics and enzymatic activity of lysozyme, Phys. Rev. E, 64 (2001) 052905.

[66] G. Schirò, A. Cupane, E. Vitrano, F. Bruni, Dielectric relaxations in confined hydrated myoglobin, J. Phys. Chem. B, 113 (2009) 9606-9613.

[67] G.P. Johari, E. Whalley, The dielectric properties of ice Ih in the range 272-133 K, J. Chem. Phys., 75 (1981) 1333-1340.

[68] R. Biehl, B. Hoffmann, M. Monkenbusch, P. Falus, S. Préost, R. Merkel, D. Richter, Direct observation of correlated interdomain motion in alcohol dehydrogenase, Phys. Rev. Lett., 101 (2008) 138102. 
[69] R. Biehl, M. Monkenbusch, D. Richter, Exploring internal protein dynamics by neutron spin echo spectroscopy, Soft Matter, 7 (2011) 1299-1307.

[70] R. Inoue, R. Biehl, T. Rosenkranz, J. Fitter, M. Monkenbusch, A. Radulescu, B. Farago, D. Richter, Large domain fluctuations on 50-ns timescale enable catalytic activity in phosphoglycerate kinase, Biophys. J., 99 (2010) 2309-2317.

[71] J. Lal, P. Fouquet, M. Maccarini, L. Makowski, Neutron spin-echo studies of hemoglobin and myoglobin: multiscale internal dynamics, J. Mol. Biol., 397 (2010) 423-435.

[72] N. Smolin, R. Biehl, G.R. Kneller, D. Richter, J.C. Smith, Functional domain motions in proteins on the 1-100 ns timescale: comparison of neutron spin-echo spectroscopy of phosphoglycerate kinase with molecular-dynamics simulation, Biophys. J., 102 (2012) 1108-1117.

[73] Y. Miyazaki, T. Matsuo, H. Suga, Low-Temperature Heat Capacity and Glassy Behavior of Lysozyme Crystal, J. Phys. Chem. B, 104 (2000) 8044-8052.

[74] W. Doster, A. Bachleitner, R. Dunau, M. Hiebl, E. Lüscher, Thermal properties of water in myoglobin crystals and solutions at subzero temperatures, Biophys. J., 50 (1986) 213-219.

[75] F. Demmel, W. Doster, W. Petry, A. Schulte, Vibrational frequency shifts as a probe of hydrogen bonds: thermal expansion and glass transition of myoglobin in mixed solvents, Eur. Biophys. J., 26 (1997) 327-335.

[76] S. Khodadadi, J.H. Roh, A. Kisliuk, E. Mamontov, M. Tyagi, S.A. Woodson, R.M. Briber, A.P. Sokolov, Dynamics of Biological Macromolecules: Not a Simple Slaving by Hydration Water, Biophys. J., 98 (2010) 1321-1326.

[77] V.N. Morozov, T.Y. Morozova, Viscoelastic properties of protein crystals: triclinic crystals of hen egg white lysozyme in different conditions, Biopolymers, 20 (1981) 451-467.

[78] A. Panagopoulou, A. Kyritsis, R. Sabater i Serra, J.L. Gómez Ribelles, N. Shinyashiki, P. Pissis, Glass transition and dynamics in BSA-water mixtures over wide ranges of composition studied by thermal and dielectric techniques, Biochim. Biophys. Acta, 1814 (2011) 1984-1996.

[79] J. Swenson, H. Jansson, R. Bergman, Relaxation processes in supercooled confined water and implications for protein dynamics, Phys. Rev. Lett., 96 (2006) 247802.

[80] C.A. Angell, Relaxation in liquids, polymers and plastic crystals — strong/fragile patterns and problems, J. Non-Cryst. Solid., 131-133 (1991) 13-31.

[81] J.L. Green, J. Fan, C.A. Angell, The protein-glass analogy: New insight from homopeptide comparisons, J. Phys. Chem., 98 (1994) 13780-13790.

[82] S. Capaccioli, K.L. Ngai, Resolving the controversy on the glass transition temperature of water?, J. Chem. Phys., 135 (2011) 104504.

[83] D.E. Shaw, P. Maragakis, K. Lindorff-Larsen, S. Piana, R.O. Dror, M.P. Eastwood, J.A. Bank, J.M. Jumper, J.K. Salmon, Y. Shan, W. Wriggers, Atomic-Level Characterization of the Structural Dynamics of Proteins, Science, 330 (2010) 341-346.

[84] D.N. LeBard, D.V. Matyushov, Ferroelectric Hydration Shells around Proteins: Electrostatics of the Protein-Water Interface, J. Phys. Chem. B, 114 (2010) 9246-9258. 